\title{
BMJ Open Low-dose prednisolone treatment of early rheumatoid arthritis and late cardiovascular outcome and survival: 10-year follow-up of a 2-year randomised trial
}

\author{
Sofia Ajeganova, ${ }^{1}$ Björn Svensson, ${ }^{2}$ Ingiäld Hafström, ${ }^{1}$ on behalf of the \\ BARFOT Study Group
}

To cite: Ajeganova S, Svensson B, Hafström I, et al. Low-dose prednisolone treatment of early rheumatoid arthritis and late cardiovascular outcome and survival: 10-year follow-up of a 2-year randomised trial. BMJ Open 2014;4:e004259. doi:10.1136/bmjopen-2013004259

- Prepublication history for this paper is available. To view please visit the journal (http://dx.doi.org/10.1136/ bmjopen-2013-004259).

Received 15 October 2013 Revised 13 March 2014 Accepted 17 March 2014

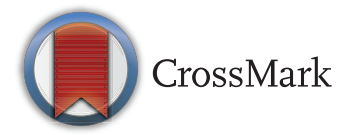

${ }^{1}$ Rheumatology Unit, Department of Medicine, Karolinska Institutet at Karolinska University Hospital Huddinge, Stockholm, Sweden

${ }^{2}$ Department of Clinical Sciences, Section of Rheumatology, Lund University, Lund, Sweden

Correspondence to Dr Sofia Ajeganova; sofia. ajeganova@karolinska.se

\section{ABSTRACT}

Objective: To examine the long-term effects of early low-dose prednisolone use in patients with rheumatoid arthritis (RA) on cardiovascular (CV) morbidity and mortality.

Design: Retrieval of data from a 2-year open randomised trial comparing prednisolone $7.5 \mathrm{mg} /$ day in addition to disease-modifying antirheumatic drugs (DMARDs) with DMARD therapy alone. Participants were followed for 10 years since inclusion into the original prednisolone trial or until occurrence of the studied outcomes.

Setting: Secondary level of care; six participating centres from southern Sweden; both urban and rural populations.

Participants: Overall, 223 patients with early RA were included. The participants had no history of CV events at baseline and incident cases were identified via the Swedish Hospital Discharge and Cause of Death Registries.

Outcomes: Composite CV events, that is, ischaemic coronary and cerebrovascular events, components of the composite CV outcome, and death. Relative HRs from Cox proportional-hazards regression models were calculated.

Results: Within 2041 person-years, 17 incident composite CV events occurred in 112 patients (15\%) randomised to prednisolone, and 15 events of 111 patients $(14 \%)$ who were assigned not to receive prednisolone. There were nine deaths $(8 \%)$ in each group. The age-adjusted relative hazards (HRs; $95 \%$ $\mathrm{Cl})$ for the first composite CV event, first coronary event and death in the prednisolone group versus the group not treated with prednisolone were 1.8 (0.9 to 3.6), 0.98 (0.4 to 2.6) and 1.6 (0.6 to 4.1), respectively. The risk for the first cerebrovascular event showed a 3.7 -fold increased relative hazard $(95 \% \mathrm{Cl}$ 1.2 to 11.4) among prednisolone treated patients.

Conclusions: In this inception cohort study of lowdose prednisolone use during the first 2 years of RA disease, the incidence of ischaemic coronary artery events was similar in the two treatment groups, whereas the long-term risk of ischaemic

\section{Strengths and limitations of this study}

Randomised allocation to prednisolone therapy to patients with early rheumatoid arthritis diagnosed according to the American College of Rheumatology (ACR) criteria.

- The treatment protocol was highly compliant with only a few patients who were lost to follow-up or discontinued the allocated therapy.

- Data on outcomes were derived from the reliable nationwide registry system and sampled during a long observation period sufficient for development of studied complications.

- The original prednisolone trial was not primarily designed to examine the risk of cardiovascular (CV) events and mortality; and the population studied was relatively young with a low burden of traditional CV risk factors at inclusion.

cerebrovascular events was higher in the prednisolone group. There was a trend towards reduced survival in the prednisolone group.

Trial registration number: ISRCTN20612367.

\section{INTRODUCTION}

Glucocorticoids (GCs) are powerful antiinflammatory agents which have been used since the 1950 s, first, as symptomatic treatment of rheumatoid arthritis (RA), but in the last few years as disease-modifying therapy. Thus, inhibition of the progression of radiological damage in RA has been documented for GCs given in addition to disease-modifying antirheumatic drugs (DMARDs). ${ }^{1-3}$ However, the relationship between short-term and long-term GC exposure and cardiovascular (CV) events and mortality in RA is still controversial. 
The discussion on the side effects of GC use is still far from settled. While adverse effects of long-term GCs use, at least at high doses, are well recognised in the general population and include effects on blood pressure, insulin resistance, lipid profile, homeostasis, body weight and fat distribution, ${ }^{4-10}$ the nature of unfavourable $\mathrm{CV}$ effects and possible modulation of effects of GCs by other processes in RA are less well known. ${ }^{11-13}$ Some data are available which imply that chronic GC use has no additive proatherogenic effects in the inflammatory milieu. ${ }^{14}{ }^{15}$ A systematic literature review has shown poor association between low-dose GC exposure and CV risk factors, and probably no effect on atherosclerosis in patients with RA. ${ }^{16}$ GCs may have antiatherogenic effects mediated by their anti-inflammatory and antiproliferative actions in the vessel wall, and modify the recovery from occlusive vascular events and intravascular injury. ${ }^{17-19}$

However, in the scientific literature, the potential risks and benefits of GC exposure in RA have shown disparate results. Increased $\mathrm{CV}$ and/or mortality risk associated with the use of GCs, particularly with increasing doses, has been found in several studies, ${ }^{20-25}$ while other studies have found GC use to improve $\mathrm{CV} /$ mortality prognosis ${ }^{26}$ or to have no or an uncertain effect. ${ }^{27}{ }^{28}$ Diversity of outcome definitions, different stages of RA disease, GC exposure in various dosages at any time during follow-up and the potential for confounding by indication in observational cohorts make the results of the studies not fairly conclusive.

In an attempt to shed some light on the CV risk of exposure to low-dose prednisolone in early RA, we performed this study with retrieval of data from the previously conducted randomised prednisolone trial, a BARFOT (Better Anti-Rheumatic PharmacOTherapy) study. ${ }^{3}$ In that trial, prednisolone $7.5 \mathrm{mg} /$ day added to the initial DMARD retarded the progression of radiographic damage after 2 years, provided a high remission rate and was well tolerated.

\section{PATIENTS AND METHODS}

Adults with a diagnosis of RA according to the American College of Rheumatology (ACR) classification criteria $1987^{29}$ and symptom duration $\leq 12$ months were eligible for the BARFOT inception cohort study. ${ }^{30}$ The design of the 2-year low-dose prednisolone multicentre open-label randomised study, nested in the BARFOT cohort, has been described in detail previously. ${ }^{3}$ This trial is registered with ISRCTN http://www.isrctn.org, number 20612367. Of the 250 patients who entered the study with the treatments as assigned, 27 with a history of prior CV events were excluded for the purpose of the current study. The final study population thus included 112 patients of the prednisolone randomisation arm, prednisolone group (P-group), and 111 patients of the no prednisolone arm, no-prednisolone group (NoP-group). Figure 1 shows the flow of participation in the study.
After randomisation, the patients received $7.5 \mathrm{mg}$ oral prednisolone daily in addition to their initial DMARD therapy, or DMARD therapy alone during 2 years. Violation of the 2-year protocol-specified therapy was uncommon, a total of eight cases. DMARDs were prescribed at the discretion of the treating rheumatologists who were encouraged to adhere to clinical practice guidelines.

Patients underwent standard laboratory testing for erythrocyte sedimentation rate (ESR) and $\mathrm{C}$ reactive protein (CRP), and assessment of RA disease at inclusion and after 3, 6, 12, 18 and 24 months, which included the Disease Activity Score for 28 joints (DAS28) $^{31}$ and the Swedish version of the Stanford Health Assessment Questionnaire (HAQ). ${ }^{32}$ Remission was defined according to the DAS28 remission criterion, DAS28<2.6 $6^{31}$ and good response according to the European League Against Rheumatism (EULAR) response criteria as improvement in DAS28 of at least 1.2 units and achievement of an absolute score of $<3.2{ }^{33}$

Sera from study enrolment were analysed for IgM rheumatoid factor (RF), using the Serodia agglutination test, (Fujirebio, Tokyo, Japan), positive RF defined as a titre of $>20 \mathrm{IU} / \mathrm{mL}$. Anticitrullinated peptide antibody (anti-CCP) was analysed using the ELISA CCP2 test, (Euro-Diagnostica, Malmö, Sweden), positive anti-CCP defined as a titre $>25 \mathrm{U} / \mathrm{mL}$.

Information on smoking status (ever or never), hypertension, diabetes mellitus, hyperlipidaemia, body mass index (BMI), use of prednisolone, DMARDs and biological agents from the first visit and during follow-up was obtained from the BARFOT database and from medical records.

All study participants provided written informed consent.

\section{Outcome assessment}

The CV events considered were acute myocardial infarction (AMI), hospitalisation for angina pectoris, coronary artery bypass grafting or percutaneous coronary intervention, ischaemic stroke and transient ischaemic attack (TIA).

The endpoints of the study were the time to the first CV event: the first composite event (ie, the first coronary artery or cerebrovascular event), the components of the composite event or death from all causes. CV events were defined after the International Classification of Diseases, Ninth Revision (ICD-9) and Tenth Revision (ICD-10) codes: AMI (ICD-9 410, ICD-10 I21), angina pectoris $(411,413$, I20) or coronary intervention (3066$3067,3080,3092,3105,3127,3141,3158$, Y832); ischaemic stroke and TIA (433-436, I63-I66, G45).

The observation period started between September 1995 and December 1999, that is, when the patients were included in the main BARFOT cohort. Each patient was followed for 10 years or until the occurrence of the first-ever incident CV event or death. 
Figure 1 Flow diagram for the randomisation and participation in the 2-year randomised part of the study. AMI, acute myocardial infarction; CVD, cardiovascular disease; TIA, transient ischaemic attack.

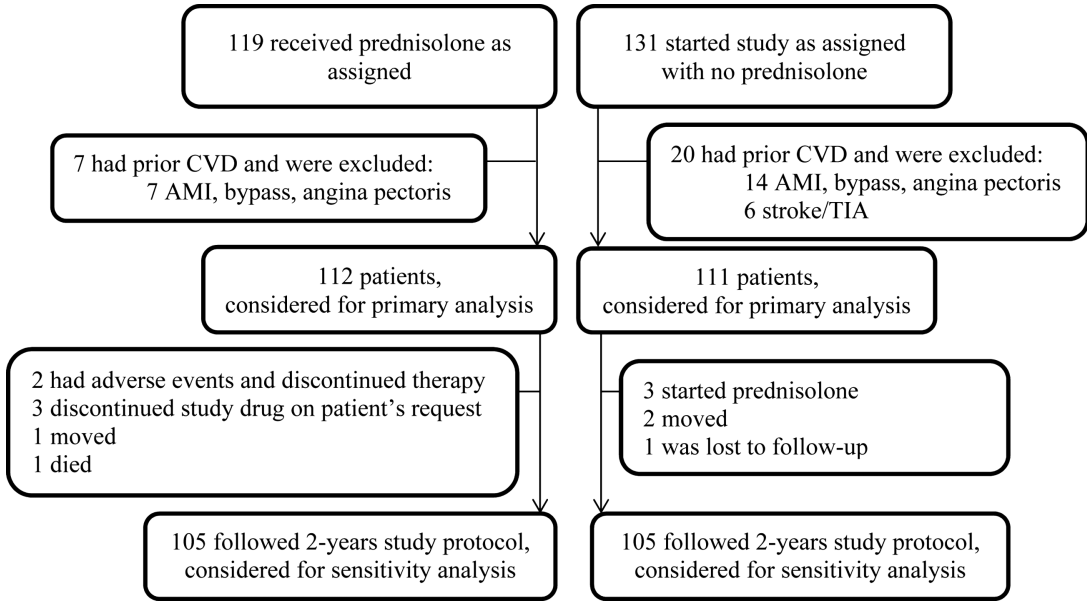

All participants could be followed through record linkage to the nationwide Causes of Death Registry and the Swedish Hospital Discharge Registry between January 1987 and December 2009. The registers used for this study have nationwide coverage and are complete, and their diagnostic validity is estimated to be high. ${ }^{34}$

\section{Statistical analysis}

The demographic and clinical features were compared using the $\mathrm{t}$ test, the Mann-Whitney $\mathrm{U}$ test, the $\chi^{2}$ or Fisher's exact tests, as suitable. Area under the curve (AUC) was calculated using the trapezoidal rule for the $\mathrm{RA}$ disease measures assessed at all time points.

If outcomes were considered to be randomly distributed in time, incidence rates (with the 95\% CI for a Poisson count) were presented as events per 100 personyears at risk.

For primary analysis, we collected and analysed endpoint data on all participants. For the time to the endpoint, we computed the Kaplan-Meier product-limit estimates of the event-free survival time and compared the randomised groups using a two-sided log-rank test. We calculated the relative HRs and 95\% CIs from the Cox proportional-hazards regression models. Covariates for adjusted Cox analyses were prespecified as variables which were imbalanced in the randomisation arms, and adjusted for in multivariate analyses if univariate tests showed significant association with the outcomes. Finally, we analysed all endpoints restricted to the participants who were adherent to the allocated intervention and clinical trial instructions as stipulated in the protocol (figure 1).

All significance tests were two tailed and conducted at the 0.05 level of significance. Statistical analyses were performed using IBM SPSS, V.20 (SPSS Inc, Chicago, Illinois, USA).

\section{RESULTS}

As shown in table 1, the treatment groups differed in age but not sex or traditional CV risk factors, except for hypertension which was less common in the P-group $(p=0.049)$. Disease characteristics and antirheumatic medications at baseline were well balanced between the study groups, but the cumulative burden of disease within 2 years after inclusion was lower in patients treated with prednisolone except for AUC-CRP, as compared with those who did not receive prednisolone.

\section{Treatments and traditional CV risk factors during follow-up}

The prednisolone therapy was assessed during follow-up to the last visit in the two study groups.

In the P-group, 59/111 patients (53\%) stopped prednisolone treatment after the first two study years. A further $30 / 109$ patients $(28 \%)$ had stopped prednisolone after a total of 4-5 years. Twenty-three of the remaining 106 patients $(22 \%)$ continued prednisolone for more than 8 years. The mean (SD) daily dose of prednisolone, in those taking prednisolone, decreased from 7.2 (1.1) $\mathrm{mg}$ at the 2-year follow-up to 6.5 (3.6) $\mathrm{mg}$ at the 4-year follow-up and 4.9 (3.3) $\mathrm{mg}$ at the 8-year assessment.

In the NoP-group, most patients (94\%) continued without prednisolone after the two study years, although prednisolone therapy was initiated and maintained over a period of more than 6 months during the follow-up in $7 / 111$ patients $(6 \%)$, with a mean average dose (SD) of 5.2 (1.4) mg daily.

Ever usage of a biological agent throughout 10 years of observation was evenly distributed in the treatment arms (15\% of the patients in each group).

The mean (SD) BMI at the 2-year visit was similar in the two study groups, 26.2 (4.8) and 27.1 (4.4) in the P-group and the NoP-group, respectively, $\mathrm{p}=0.21$. None of the enrolled patients became a smoker during the observation period. Thus, the never-smoking status throughout the study was not changed. During follow-up, the number of patients registered with hypertension increased to $33(30 \%)$ in the P-group and to 41 patients $(37 \%)$ in the NoP-group, $p=0.24$. Diabetes mellitus was recorded in 2 patients $(2 \%)$ in the P-group versus 10 patients $(9 \%)$ in the NoP-group, $\mathrm{p}=0.017$, and 
Table 1 Clinical characteristics and cardiovascular outcomes for patients randomised to prednisolone or no prednisolone treatment

\begin{tabular}{|c|c|c|c|}
\hline & \multicolumn{2}{|c|}{ Groups by randomisation } & \multirow[b]{2}{*}{ p Value } \\
\hline & Prednisolone $n=112$ & No-prednisolone $n=111$ & \\
\hline Age at inclusion, years & $50.6(14.1)$ & $56.9(13.0)$ & 0.001 \\
\hline Female, n (\%) & 77 (69) & $76(69)$ & 0.96 \\
\hline \multicolumn{4}{|l|}{ Traditional CV risk factors at baseline } \\
\hline $\mathrm{BMI}, \mathrm{kg} / \mathrm{m}^{2}$ & $25.2(4.3)$ & $26.4(4.2)$ & 0.87 \\
\hline Smoking ever, n (\%) & $73(65)$ & $64(58)$ & 0.25 \\
\hline Hypertension, n (\%) & $14(12.5)$ & $25(22.5)$ & 0.049 \\
\hline Diabetes mellitus, $\mathrm{n}(\%)$ & 0 & $4(4)$ & 0.06 \\
\hline Hyperlipidaemia, n (\%) & $1(1)$ & $1(1)$ & 1.00 \\
\hline \multicolumn{4}{|l|}{ Baseline RA characteristics } \\
\hline Disease duration, months & $6.5(3.5)$ & $5.8(2.8)$ & 0.12 \\
\hline RF positive, n (\%) & $72(65)$ & $72(65)$ & 1.00 \\
\hline Anti-CCP positive, $\mathrm{n}(\%)$ & $58(64)$ & $50(59)$ & 0.50 \\
\hline DAS28 & $5.3(1.1)$ & $5.4(1.1)$ & 0.34 \\
\hline $\mathrm{ESR}, \mathrm{mm} / \mathrm{h}$ & $38(26)$ & $37(25)$ & 0.83 \\
\hline $\mathrm{CRP}, \mathrm{mg} / \mathrm{L}$ & $22(8-51)$ & $21(8-53)$ & 0.96 \\
\hline $\mathrm{HAQ}$ & $1.0(0.6)$ & $1.0(0.7)$ & 0.58 \\
\hline \multicolumn{4}{|l|}{ Started DMARDs at baseline } \\
\hline MTX, n (\%) & $57(51)$ & $61(55)$ & 0.54 \\
\hline SSZ, n (\%) & $36(32)$ & $38(34)$ & 0.74 \\
\hline AMA, n (\%) & $9(8)$ & $4(4)$ & 0.25 \\
\hline Gold, n (\%) & $9(8)$ & $8(7)$ & 0.82 \\
\hline Ever use of biological agents during the study, $n(\%)$ & $17(15)$ & $17(15)$ & 1.00 \\
\hline \multicolumn{4}{|l|}{ Cumulative RA disease burden in the first 2 years } \\
\hline AUC-DAS28 & $71.2(28.1)$ & $89.3(28.4)$ & $<0.001$ \\
\hline AUC-ESR, mm/h & $386(254)$ & $504(348)$ & 0.011 \\
\hline AUC-CRP, mg/L & $253(189-364)$ & $296(162-480)$ & 0.37 \\
\hline AUC-HAQ & $11.5(10.9)$ & $17.6(12.7)$ & 0.001 \\
\hline \multicolumn{4}{|l|}{ Outcomes } \\
\hline Incident CV event, total, n (\%) & $17(15.2)$ & $15(13.5)$ & 0.72 \\
\hline Incident ischaemic coronary event, n (\%) & $7(6.2)$ & $10(9.0)$ & 0.44 \\
\hline Incident ischaemic cerebrovascular event, n (\%) & $10(8.9)$ & $5(4.5)$ & 0.19 \\
\hline Death, n (\%) & $9(8)$ & $9(8)$ & 0.98 \\
\hline
\end{tabular}

hyperlipidaemia in $6(5 \%)$ versus 3 patients $(3 \%)$ in the respective groups, $\mathrm{p}=0.50$.

\section{Study outcomes}

During 10 years of observation, the total number of incident CV events was 17 of 112 patients (15\%) in the P-group ( 7 cases of AMI, hospitalisation for angina pectoris and coronary interventions; 7 cases of ischaemic stroke and 3 of TIA), as compared with 15 of 111 patients $(14 \%)$ in the NoP-group (corresponding events in 10,3 and 2 cases), $\mathrm{p}=0.72$. None of the events was fatal. Incident $\mathrm{CV}$ events occurred after a median of 5.4 years (range 3-114 months) in the P-group and 4.9 years (range 2-120 months) in the NoP-group, $\mathrm{p}=0.66$.

For the entire cohort, the total follow-up time was 2041 person-years. The cumulative incidence of CV events was 1.7 per 100 person-years (95\% CI 0.9 to 2.5 ) in the P-group and 1.5 per 100 person-years (95\% CI 0.7 to 2.3) in the NoP-group. The rate of the first-ever observed ischaemic coronary event was 0.7 per 100 person-years (95\% CI 0.2 to 1.2 ) in the P-group and 1.0 per 100 person-years $(95 \%$ CI 0.4 to 1.6$)$ in the NoP-group. Occurrence of the first-ever cerebrovascular event was nominally twofold higher in the P-group, 10 cases $(8.9 \%)$, rates of 1.0 per 100 person-years $(95 \%$ CI 0.4 to 1.6$)$, as compared with the NoP-group, five cases $(4.5 \%)$, rates of 0.5 per 100 person-years $(95 \%$ CI 0.1 to $0.9)$. The pattern of distribution of the $\mathrm{CV}$ outcomes over time was even in the groups.

Nine mortalities $(8 \%)$ were observed in each group during the 10-year period.

\section{Primary analyses of outcomes}

In the univariate Cox proportional hazard models, age at the study inclusion was found to be associated with 
the incident composite CV event, HR 1.08 (95\% CI 1.05 to 1.12), but not hypertension at inclusion, or AUCs of DAS28, ESR and HAQ during the first 2 years of RA disease. Similar results were obtained in the univariate analyses of the CV subgroups and death, data not shown.

After adjustment for age, the relative hazards for the composite CV event endpoint and death did not differ statistically significantly between the P-group and the NoP-group, HRs (95\% CI) 1.8 (0.9 to 3.6), and 1.6 (0.6 to 4.1 ), respectively (figure $2 \mathrm{~A}, \mathrm{D}$ ). When analysing the components of the composite $\mathrm{CV}$ event endpoint, the hazard for the first coronary event was much the same in the two groups, HR 0.98 (0.4 to 2.6), while the hazard for the first cerebrovascular event among prednisolone treated patients was 3.7 times (95\% CI 1.2 to 11.4) higher than that among those who did not receive prednisolone (figure 2B,C).

Incident Composite Cardiovascular Event End Point

A

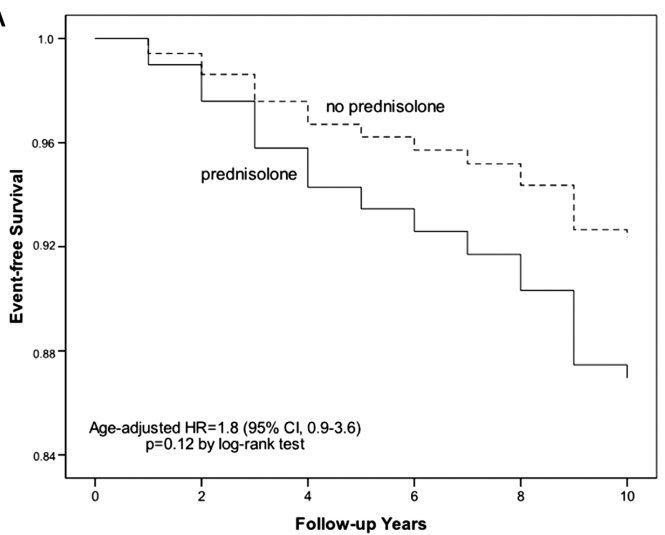

No. At Risk

NoP-group $111(1) \quad 110(5) \quad 104(3) \quad 98(2) \quad 94(3) \quad 46(1)$

$\begin{array}{llllll}\text { P-group } & 112(2) \quad 109(4) & 104(3) & 100(2) \quad 96(6) & 44(0)\end{array}$

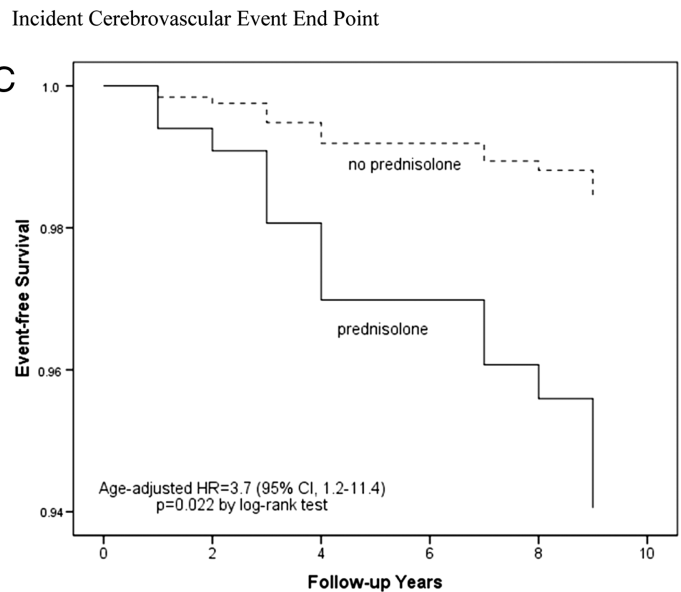

No. At Risk

NoP-group $111(1) \quad 109(2) \quad 103(2) \quad 97(0) \quad 93(0) \quad 46(0)$

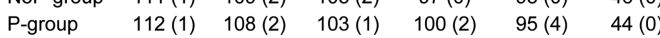

Shown are the Kaplan-Meier curves comparing the P-group with the NoP-group for the time to the first composite CV event (figure 2A), the first-ever ischaemic coronary artery event (figure 2B), the first-ever cerebrovascular event (figure 2C) and death (figure 2D). The relative age-adjusted HRs were calculated with the use of a Cox proportional-hazards model. The tables below the panels represent the number of participants at risk for the endpoints (number of the endpoints) at 2-year intervals throughout the 10-year observation.

\section{Sensitivity analyses of outcomes}

When we considered data for the patients who followed the assigned treatment, 105 participants in each randomisation arm, the results were not changed. Given the decreasing survival trend 5 years after the start of the study, we recalculated estimates for the time to death between 5 and 10 years after enrolment. The additional

Incident Ischemic Coronary Artery Event End Point
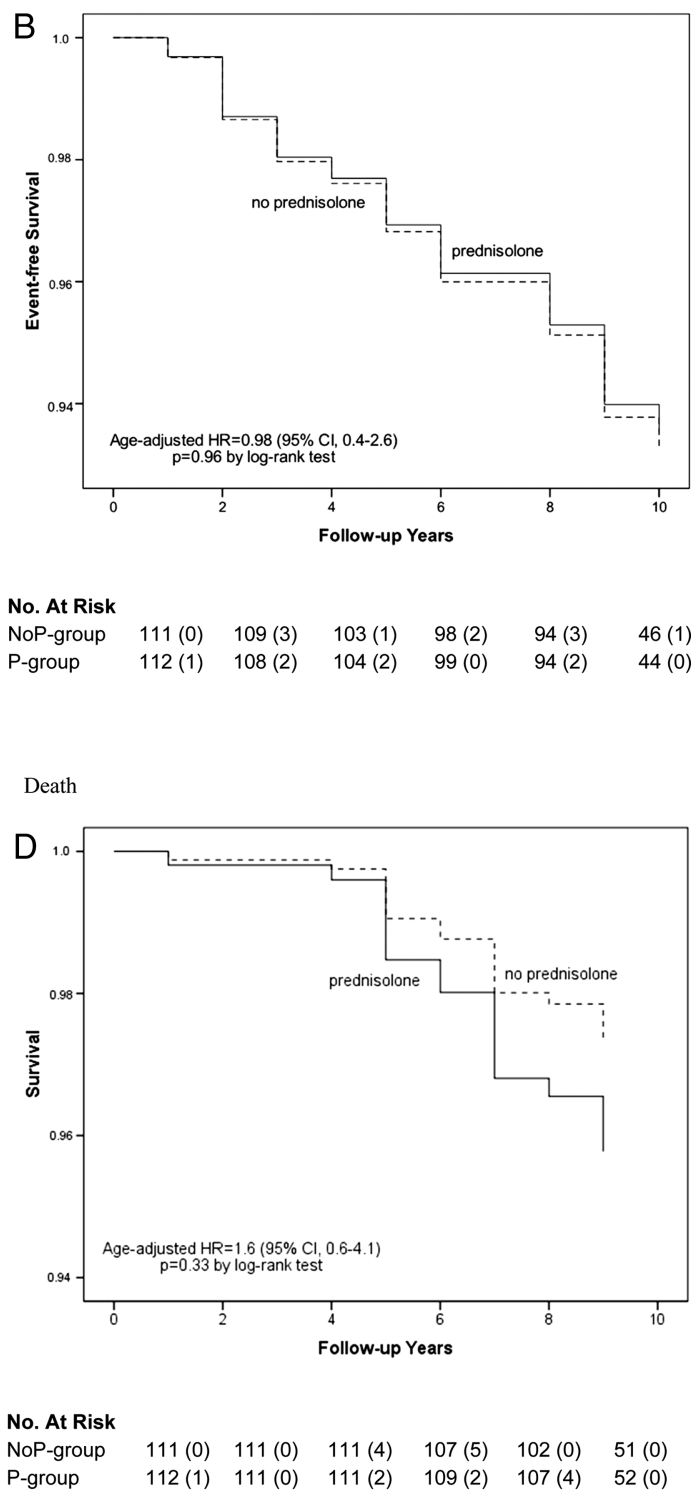

Figure 2 (A-D) Primary analysis of the study outcomes. NoP-group, no-prednisolone group; P-group, prednisolone group. 
analysis for this time period comparing the relative age-adjusted hazards for death in the P-group with the NoP-group yielded stable findings similar to that for the whole 10-year follow-up, 1.4 (95\% CI 0.5 to $3.9, \mathrm{p}=0.47$ ).

\section{Further analyses of outcomes in relation to therapy response}

We performed analyses in order to investigate whether the effect of prednisolone was mediated through reduced inflammation. The cohort was stratified by therapy response at the 2-year visit according to DAS28 remission criteria and EULAR response criteria.

Relative age-adjusted hazards for death among participants with DAS28 remission tended to be lower than those among patients who did not achieve DAS28 remission. The HRs were 0.41 (95\% CI 0.15 to 1.15 ), $p=0.089$ in the whole cohort, 0.30 ( 0.07 to 1.19 ), $\mathrm{p}=0.087$ in the $\mathrm{P}$-group and 0.42 (0.09 to 2.03), $\mathrm{p}=0.28$ in the NoP-group.

A similar tendency to decreased estimated relative mortality risks was observed among good EULAR responders compared with those not achieving good response. The age-adjusted relative hazards were 0.37 ( 0.15 to 0.95$), \mathrm{p}=0.038$ in the whole cohort, $0.45(0.12$ to 1.70$), \mathrm{p}=0.24$ in the P-group and 0.28 ( 0.07 to 1.13 ), $\mathrm{p}=0.074$ in the NoP-group.

These relative differences were sustained over time. As to the composite $\mathrm{CV}$ event endpoint, stratifying by therapy efficacy did not show differences or trends to differences in the risks for the outcome, data not shown.

\section{DISCUSSION}

We presented herein the results of the 10-year follow-up of CV events and deaths in a large, multicentre, prospective, open-label, randomised clinical trial of low-dose prednisolone, $7.5 \mathrm{mg} /$ day, over the first 2 years of early RA disease in patients with no previous GC or DMARD therapy. The estimated risk for a composite $\mathrm{CV}$ event, a first coronary artery event and death did not differ between patients assigned to receive prednisolone or not. However, the observed age-adjusted relative risk for a first cerebrovascular event among prednisolone treated patients was increased by about four times that among those not treated with prednisolone. It should be noticed, however, that due to the low number of events and the wide estimated rates, a clinically important CV risk in the long term in an individual patient treated with prednisolone is uncertain.

To the best of our knowledge, this is the first study addressing $\mathrm{CV}$ and survival consequences of exposure to low-dose prednisolone during 2 years in early RA in a randomised design. It should be mentioned that a good half of those randomised to prednisolone stopped that treatment after 2 years from baseline and that a further fourth quarter stopped after 4-5 years. In contrast, only a few patients who were randomised not to take prednisolone initiated prednisolone therapy during follow-up. Still, the distribution of traditional risk factors during the study period (BMI, hypertension and hyperlipidaemia) was similar in the treatment groups, which strengthens the present findings and also challenges the idea of a negative impact of prednisolone on traditional $\mathrm{CV}$ risk factors in patients with an inflammatory condition.

Our study is different in several important ways to previous studies in respect of the relationship between GC exposure and $\mathrm{CV}$ in RA and these differences make a broad comparison of the results difficult.

Based on prescription databases from the general population, Wei $e t a l^{35}$ reported an increased risk for $\mathrm{CV}$ disease in GC users only in doses $\geq 7.5 \mathrm{mg}$ of prednisolone or equivalent, the relative risk being 2.56 (CI 2.18 to 2.99). Avina-Zubieta et $a l^{22}$ determined that the current dose ( $13 \%$ risk increase per additional $5 \mathrm{mg} /$ day), cumulative duration of past GC use (10\% risk increase for every additional year) and total cumulative dose $(6 \%$ per each gram accumulated in the past) were independently associated with an increased risk of first myocardial infarction (MI) in RA cases identified through administrative data sources. In that study, GC exposure again included GCs dispensed by pharmacists at some time over the entire disease course. It should also be noted that participants using GC had a high absolute risk of the $\mathrm{CV}$ outcomes and, owing to the lack of direct information on individual patient characteristics, surrogate indices were applied to control for confounding by indication. In a population-based incidence RA cohort, Davis et $a l^{25}$ reported that, overall, a higher risk of the initial combined $\mathrm{CV}$ outcome was associated with recent exposure to GC, adjusted HR 1.66 (95\% CI 1.14 to 2.41 ), and the highest tertile of cumulative exposure ( $>7000 \mathrm{mg}$ of prednisone equivalents), HR 2.11 (95\% CI 1.47 to 3.04), whereas there was no association with past GC exposure $>3$ months, or mid tertile cumulative exposure (>1500 to $\leq 7000 \mathrm{mg}$ ).

Conversely, a lower prevalence for lifetime CV morbidity among patients with prolonged exposure to GCs, similar to conventional DMARDs and anti-tumour necrosis factor blockers, has been shown by Naranjo et $a l^{36}$ in the cross-sectional study in non-selected outpatients with RA, HR 0.95 (95\% CI 0.92 to 0.98 ). In a prospective RA cohort, Gonzalez-Gay et $a l^{37}$ could not confirm an excess risk of incident $\mathrm{CV}$ events or $\mathrm{CV}$ mortality induced by a median dose of prednisone $5 \mathrm{mg}$ /day for at least 1 year, and the mean (SD) cumulative dose of prednisone of 13.5 (9) $\mathrm{g}$ at the end of the study. Interestingly, risk of hospitalisation for MI and stroke, which was assessed in the nested case-control study reported by Solomon et $a l,{ }^{24}$ was significantly increased only if GC were used in monotherapy (MTX monotherapy used as the reference group), but not in combination with other DMARDs.

Our results further suggest that it may be possible to avoid the potential negative effects of GC on future ischaemic coronary artery complications and survival prognosis if GCs are used in low doses, over limited 
time, in conjunction with DMARDs in patients with a low baseline rate of traditional CV risk factors. This is in line with the encouraging reports about associations between effective antirheumatic therapy and favourable overall CV and survival prognosis, ${ }^{38-41}$ which formed the basis for the hypothesis that a dampening of systemic inflammation by GC may halt development of atherosclerotic disease.

Using the present knowledge, it seems fair to say that even if the possibility of an excess CV risk associated with GC exposure cannot be excluded, the negative effects of GC may be counteracted by the positive impact of suppression of chronic inflammation. Thus, the significant benefits of the low-dose GC therapy, both in terms of disease activity and radiographic progression in the present trial, ${ }^{2} 3$ as compared with therapy not including GC, could most likely have overcome adverse coronary events and overall survival. In support of this hypothesis, the previous subgroup analysis has shown that a low dose of prednisolone in early RA was not proatherogenic, considering the carotid artery intimamedia measures, presence of atherosclerotic plaques and endothelial function after 5 years of follow-up. ${ }^{42}$ Accordingly, we observed a tendency towards reduction in mortality for patients with a better therapy response, possibly due to an overall decrease in inflammation related to the antirheumatic therapy.

When it comes to the potential cerebrovascular risk of GC use, the evidence is limited. There are studies showing an excess risk when using GC, ${ }^{28} 35$ while several others have failed to find any excess risk. ${ }^{43}{ }^{44}$ Notably, few studies have divided CV events into subtypes. Such a subdivision could be of importance, considering the possibility of different aetiologies of coronary and cerebrovascular events. The results of our study indicate that even low-dose GC exposure could be associated with long-term cerebrovascular safety issues in patients with RA. What pathways of the GC would be involved in occurrence of cerebrovascular events but not coronary events are unclear. About $14-30 \%$ of all strokes in the general population are of cardioembolic origin, and atrial fibrillation is a risk factor for this type of ischaemic stroke. ${ }^{45}$ An almost twofold increased risk of atrial fibrillation or flutter has been reported in current and longterm users of GC in a population-based case-control study. ${ }^{46}$ As we found differences in the risk of ischaemic coronary and cerebrovascular complications, we cannot exclude that the excess cerebrovascular risk in our study was essentially confined to participants with other specific underlying risk factors, for example, atrial fibrillation. Such a possibility, however, could not be tested here through hospitalisation registries.

Our study has several important strengths such as an incidence cohort of patients with RA with the diagnosis validated by the ACR criteria, randomised allocation to therapy within the setting of prospective follow-up, long observation period sufficient for development of studied complications, high compliance with the treatment protocol, few losses to follow-up or therapy discontinuation. Furthermore, the reliable nationwide registry system ascertains the outcomes.

However, these data should be interpreted with caution because it is not clear to what extent a more prolonged therapy with oral GCs may affect the risk of CV-related adverse clinical events and survival. Further, the population of this study was relatively young and had a low frequency of baseline traditional CV risk factors. Then, we could not have anticipated the observed baseline imbalance between the treatment groups, but the age difference was adjusted for in the analyses. It should also be acknowledged that the prednisolone trial was not primarily designed to examine the risk of incident CV events. Given the low precision of estimated hazards and restriction of measurements to prednisolone exposure only in the first 2 years, regardless of subsequent prednisolone use, the results should be interpreted as non-definitive regarding the possible long-term risks.

\section{CONCLUSION}

This study has focused on the long-term CV and mortality risks of low-dose GC therapy in RA and adds further weight to the arguments for appropriate use of GCs in early disease. Our data suggest that the 2-year low-dose GC exposure in patients with early RA may have affected the risk of cerebrovascular events. On the other side, the results of this study would argue against GC-induced risk of ischaemic coronary artery complications in a population with low frequency of background traditional CV risk factors.

Acknowledgements The authors would like to thank the following members of the BARFOT (Better Anti-Rheumatic PharmacOTherapy) study group: Maria Andersson, Kristina Forslind, Catharina Keller, Ido Leden, Bengt Lindell, Ingemar Petersson, Christoffer Schaufelberger, Annika Teleman and Jan Theander.

Contributors $\mathrm{IH}$ and BS designed the original low-dose prednisolone study and were involved in acquisition of data. SA was responsible for the current study concept, data acquisition, statistical analyses and drafting of the manuscript. IH participated in the interpretation of data and manuscript preparation. All authors have critically revised and approved the final version of the manuscript to be published, and contributed to the study with regard to important intellectual content.

Funding This work was supported by grants from the Swedish Rheumatism Association, Stiftelsen för Rörelsehindrade i Skåne and the Regional agreement on medical training and clinical research (ALF) between the Stockholm County Council and the Karolinska Institutet.

Competing interests None.

Patient consent Obtained.

Ethics approval The study was approved by the Regional Ethic Committee, and was performed in accordance with the Declaration of Helsinki.

Provenance and peer review Not commissioned; externally peer reviewed.

Data sharing statement No additional data are available.

Open Access This is an Open Access article distributed in accordance with the Creative Commons Attribution Non Commercial (CC BY-NC 3.0) license, which permits others to distribute, remix, adapt, build upon this work noncommercially, and license their derivative works on different terms, provided 
the original work is properly cited and the use is non-commercial. See: http:// creativecommons.org/licenses/by-nc/3.0/

\section{REFERENCES}

1. Kirwan JR, Bijlsma JW, Boers M, et al. Effects of glucocorticoids on radiological progression in rheumatoid arthritis. Cochrane Database Syst Rev 2007;(1):CD006356.

2. Hafstrom I, Albertsson K, Boonen A, et al. Remission achieved after 2 years treatment with low-dose prednisolone in addition to disease-modifying anti-rheumatic drugs in early rheumatoid arthritis is associated with reduced joint destruction still present after 4 years: an open 2-year continuation study. Ann Rheum Dis 2009;68:508-13.

3. Svensson B, Boonen A, Albertsson K, et al. Low-dose prednisolone in addition to the initial disease-modifying antirheumatic drug in patients with early active rheumatoid arthritis reduces joint destruction and increases the remission rate: a two-year randomized trial. Arthritis Rheum 2005:52:3360-70.

4. Andrews RC, Walker BR. Glucocorticoids and insulin resistance: old hormones, new targets. Clin Sci (Lond) 1999;96:513-23.

5. Whitworth JA, Mangos GJ, Kelly JJ. Cushing, cortisol, and cardiovascular disease. Hypertension 2000;36:912-16.

6. Maxwell SR, Moots RJ, Kendall MJ. Corticosteroids: do they damage the cardiovascular system? Postgrad Med J 1994;70:863-70.

7. Sholter DE, Armstrong PW. Adverse effects of corticosteroids on the cardiovascular system. Can J Cardiol 2000;16:505-11.

8. Brotman DJ, Girod JP, Garcia MJ, et al. Effects of short-term glucocorticoids on cardiovascular biomarkers. J Clin Endocrinol Metab 2005;90:3202-8.

9. Brotman DJ, Girod JP, Posch A, et al. Effects of short-term glucocorticoids on hemostatic factors in healthy volunteers. Thromb Res 2006;118:247-52.

10. Girod JP, Brotman DJ. Does altered glucocorticoid homeostasis increase cardiovascular risk? Cardiovasc Res 2004;64:217-26.

11. Panoulas VF, Douglas KM, Milionis HJ, et al. Prevalence and associations of hypertension and its control in patients with rheumatoid arthritis. Rheumatology 2007;46:1477-82.

12. Dessein $\mathrm{PH}$, Joffe BI, Stanwix AE, et al. Glucocorticoids and insulin sensitivity in rheumatoid arthritis. J Rheumatol 2004;31:867-74.

13. Da Silva JA, Jacobs JW, Kirwan JR, et al. Safety of low dose glucocorticoid treatment in rheumatoid arthritis: published evidence and prospective trial data. Ann Rheum Dis 2006;65:285-93.

14. Toms TE, Panoulas VF, Douglas KM, et al. Lack of association between glucocorticoid use and presence of the metabolic syndrome in patients with rheumatoid arthritis: a cross-sectional study. Arthritis Res Ther 2008;10:R145.

15. Hoes JN, van der Goes MC, van Raalte DH, et al. Glucose tolerance, insulin sensitivity and beta-cell function in patients with rheumatoid arthritis treated with or without low-to-medium dose glucocorticoids. Ann Rheum Dis 2011;70:1887-94.

16. Ruyssen-Witrand A, Fautrel B, Saraux A, et al. Cardiovascular risk induced by low-dose corticosteroids in rheumatoid arthritis: a systematic literature review. Joint Bone Spine 2011;78:23-30.

17. Rab ST, King SB III, Roubin GS, et al. Coronary aneurysms after stent placement: a suggestion of altered vessel wall healing in the presence of anti-inflammatory agents. J Am Coll Cardiol 1991;18:1524-8.

18. Versaci F, Gaspardone A, Tomai F, et al. Immunosuppressive therapy for the prevention of restenosis after coronary artery stent implantation (IMPRESS Study). J Am Coll Cardiol 2002;40:1935-42.

19. Walker BR. Glucocorticoids and cardiovascular disease. Eur J Endocrinol 2007;157:545-59.

20. Wolfe $\mathrm{F}$, Michaud $\mathrm{K}$. The risk of myocardial infarction and pharmacologic and nonpharmacologic myocardial infarction predictors in rheumatoid arthritis: a cohort and nested case-control analysis. Arthritis Rheum 2008;58:2612-21.

21. Avina-Zubieta JA, Choi HK, Rahman MM. Corticosteroids increase risk for acute myocardial infarction in rheumatoid arthritis: the effects of careful modeling of cumulative exposure. Arthritis Rheum 2009;60:S149.

22. Avina-Zubieta JA, Abrahamowicz M, De Vera MA, et al. Immediate and past cumulative effects of oral glucocorticoids on the risk of acute myocardial infarction in rheumatoid arthritis: a population-based study. Rheumatology 2013;52:68-75.

23. Suissa S, Bernatsky S, Hudson M. Antirheumatic drug use and the risk of acute myocardial infarction. Arthritis Rheum 2006;55:531-6.
24. Solomon DH, Avorn J, Katz JN, et al. Immunosuppressive medications and hospitalization for cardiovascular events in patients with rheumatoid arthritis. Arthritis Rheum 2006;54:3790-8.

25. Davis JM III, Maradit Kremers H, Crowson CS, et al. Glucocorticoids and cardiovascular events in rheumatoid arthritis: a population-based cohort study. Arthritis Rheum 2007;56:820-30.

26. van Tuyl LH, Boers M, Lems WF, et al. Survival, comorbidities and joint damage 11 years after the COBRA combination therapy trial in early rheumatoid arthritis. Ann Rheum Dis 2010;69:807-12.

27. Wallberg-Jonsson S, Ohman ML, Dahlqvist SR. Cardiovascular morbidity and mortality in patients with seropositive rheumatoid arthritis in Northern Sweden. J Rheumatol 1997;24:445-51.

28. Nadareishvili Z, Michaud K, Hallenbeck JM, et al. Cardiovascular, rheumatologic, and pharmacologic predictors of stroke in patients with rheumatoid arthritis: a nested, case-control study. Arthritis Rheum 2008;59:1090-6.

29. Arnett FC, Edworthy SM, Bloch DA, et al. The American Rheumatism Association 1987 revised criteria for the classification of rheumatoid arthritis. Arthritis Rheum 1988;31:315-24.

30. Svensson B, Schaufelberger C, Teleman A, et al. Remission and response to early treatment of RA assessed by the Disease Activity Score. BARFOT study group. Better Anti-rheumatic Farmacotherapy. Rheumatology (Oxford) 2000;39:1031-6.

31. Prevoo ML, van't Hof MA, Kuper HH, et al. Modified disease activity scores that include twenty-eight-joint counts. Development and validation in a prospective longitudinal study of patients with rheumatoid arthritis. Arthritis Rheum 1995;38:44-8.

32. Ekdahl C, Eberhardt $\mathrm{K}$, Andersson SI, et al. Assessing disability in patients with rheumatoid arthritis. Use of a Swedish version of the Stanford Health Assessment Questionnaire. Scand J Rheumatol 1988;17:263-71.

33. van Gestel AM, Haagsma CJ, van Riel PL. Validation of rheumatoid arthritis improvement criteria that include simplified joint counts. Arthritis Rheum 1998;41:1845-50.

34. Ludvigsson JF, Andersson E, Ekbom A, et al. External review and validation of the Swedish national inpatient register. BMC Public Health 2011;11:450.

35. Wei L, MacDonald TM, Walker BR. Taking glucocorticoids by prescription is associated with subsequent cardiovascular disease. Ann Intern Med 2004;141:764-70.

36. Naranjo A, Sokka T, Descalzo MA, et al. Cardiovascular disease in patients with rheumatoid arthritis: results from the QUEST-RA study. Arthritis Res Ther 2008;10:R30.

37. Gonzalez-Gay MA, Gonzalez-Juanatey C, Lopez-Diaz MJ, et al. HLA-DRB1 and persistent chronic inflammation contribute to cardiovascular events and cardiovascular mortality in patients with rheumatoid arthritis. Arthritis Rheum 2007:57:125-32.

38. Micha R, Imamura F, Wyler von Ballmoos M, et al. Systematic review and meta-analysis of methotrexate use and risk of cardiovascular disease. Am J Cardiol 2011;108:1362-70.

39. Barnabe C, Martin BJ, Ghali WA. Systematic review and meta-analysis: anti-tumor necrosis factor alpha therapy and cardiovascular events in rheumatoid arthritis. Arthritis Care Res (Hoboken) 2011;63:522-9.

40. Westlake SL, Colebatch AN, Baird J, et al. Tumour necrosis factor antagonists and the risk of cardiovascular disease in patients with rheumatoid arthritis: a systematic literature review. Rheumatology (Oxford) 2011:50:518-31.

41. Dixon WG, Watson KD, Lunt M, et al. Reduction in the incidence of myocardial infarction in patients with rheumatoid arthritis who respond to anti-tumor necrosis factor alpha therapy: results from the British Society for Rheumatology Biologics Register. Arthritis Rheum 2007;56:2905-12.

42. Hafstrom I, Rohani M, Deneberg S, et al. Effects of low-dose prednisolone on endothelial function, atherosclerosis, and traditional risk factors for atherosclerosis in patients with rheumatoid arthritisa randomized study. J Rheum 2007;34:1810-16.

43. Avina-Zubieta JA, Abrahamowicz M, Choi HK, et al. Risk of cerebrovascular disease associated with the use of glucocorticoids in patients with incident rheumatoid arthritis: a population-based study. Ann Rheum Dis 2011;70:990-5.

44. Souverein PC, Berard A, Van Staa TP, et al. Use of oral glucocorticoids and risk of cardiovascular and cerebrovascular disease in a population based case-control study. Heart 2004:90:859-65.

45. Arboix A, Alio J. Cardioembolic stroke: clinical features, specific cardiac disorders and prognosis. Curr Cardiol Rev 2010;6:150-61.

46. Christiansen CF, Christensen S, Mehnert F, et al. Glucocorticoid use and risk of atrial fibrillation or flutter: a population-based, case-control study. Arch Intern Med 2009;169:1677-83. 